\title{
Multilinguales
}

\section{Du prêtre au traître : destins croisés du prélat par Mongo Beti et Ramon J. Sender}

From the Priest to the Betrayer: Crossed Destinies of the Prelate by Mongo Bet and Ramón J. Sender

Ebé Mathurin Ovono

\section{OpenEdition}

\section{Journals}

Édition électronique

URL : http://journals.openedition.org/multilinguales/1378

DOI : $10.4000 /$ multilinguales. 1378

ISSN : 2335-1853

Éditeur

Université Abderrahmane Mira - Bejaia

Édition imprimée

Date de publication : 1 juin 2015

Pagination : 223-237

ISSN : 2335-1535

Référence électronique

Ebé Mathurin Ovono, «Du prêtre au traître: destins croisés du prélat par Mongo Beti et Ramon J. Sender », Multilinguales [En ligne], 5 | 2015, mis en ligne le 01 juin 2015, consulté le 17 septembre 2019. URL : http://journals.openedition.org/multilinguales/1378; DOI : 10.4000/multilinguales.1378

Ce document a été généré automatiquement le 17 septembre 2019.

\section{cc) (†)}

Multilinguales est mise à disposition selon les termes de la Licence Creative Commons Attribution -

Pas d'Utilisation Commerciale - Pas de Modification 4.0 International 


\section{Du prêtre au traître : destins croisés du prélat par Mongo Beti et Ramon J. Sender}

From the Priest to the Betrayer: Crossed Destinies of the Prelate by Mongo Bet and Ramón J. Sender

Ebé Mathurin Ovono

1 Les questions religieuses ont toujours préoccupé certains écrivains, quelle que soit leur époque et quelle que soit leur aire culturelle. Dans la sphère chrétienne, la publication récente de Le Christ selon l'Afrique de Calixte Béyala ${ }^{1}$ témoigne de la pérennité de cet intérêt. Cependant, le traitement littéraire de ce thème témoigne, en général, d'un parti pris de l'écrivain. Nous sommes alors en droit de nous posons la question de savoir si la représentation de la religion dans la littérature en général et dans un roman en particulier va dans le sens de son apologie ou dans celui de sa condamnation. Nous chercherons la réponse dans la comparaison de deux romans : Le Pauvre Christ de Bomba (1956) du Camerounais Mongo Beti et dans Réquiem por un campesino español ${ }^{2}$ (1960) de l'Espagnol Ramón J. Sender.

Le choix de ces deux textes se justifie d'abord par leur ancrage dans le catholicisme ; et par leur croisement, nous montrerons que les agissements du prêtre sont les mêmes, qu'on soit en Europe ou en Afrique, qu'on soit en Espagne pendant la Guerre Civile de 1936-1939 ou au Cameroun à l'époque coloniale.

Ainsi tenterons-nous de porter un regard croisé sur le prélat, figure centrale du catholicisme dans ces deux romans, en nous concentrant sur ses rapports avec le pouvoir. Nous articulerons notre réflexion autour de quatre axes principaux: le premier portera sur les invariants des deux romans, le second sur leur titrologie, le troisième sur les relations entre l'Église et le Pouvoir et, enfin, le quatrième sur les rapports qu'entretient le prêtre avec ses fidèles. 


\section{Les invariants dans Le Pauvre Christ de Bomba et Requiem por un campesino español}

2 Le Pauvre Christ de Bomba et Réquiem por un campesino español sont deux romans formellement différents, mais thématiquement semblables. Le roman de Mongo Beti (2011) est un volume de trois cents cinquante deux (352) pages réparties en deux parties. La première est composée de huit (8) chapitres ayant pour titre chaque étape de la tournée du Révérend Père Supérieur (R.P.S.), à savoir: «Mission catholique de Bomba », "Mombet », "Timbo », "Kota», "Bitie», "Evindi», "Ekokot» et "Ekokot». La deuxième comprend treize (13) chapitres et un (1) épilogue, avec le même système titulaire que la première: "Ndimi», «Zibi», «Akamba», «Teba », «Kondo», «Kouma », "Mission catholique de Bomba », «Bomba », «Bomba», «Bomba», «Bomba », «Bomba » et « Sogolo » qui constitue l'épilogue du récit.

Le roman de Ramón J. Sender est un parangon de littérature en direct. Il se présente comme un ensemble de cent huit (108) pages dont on peut dégager la structuration en vingt et une (21) séquences (Rodríguez Gutiérrez, $2005: 11$ ).

Sa forme, de par son inconstance, est différente de son fond qui révèle un certain nombre d'invariants, au sens de la littérature comparée : «l'élément universel, 'commun', de la littérature et de la pensée littéraire » (Marino, 1979 : 323).

3 Le premier invariant dans les deux romans est la thématique de la religion symbolisée par le prêtre entouré de ses fidèles. Dans Le Pauvre Christ de Bomba, Mongo Beti met en scène un prêtre en mission d'évangélisation en Afrique Noire coloniale, tandis que Ramón J. Sender présente un prêtre en Espagne pendant la guerre civile dans Réquiem por un campesino español. Les deux personnages sont pris entre la soumission au pouvoir administratif et la protection des fidèles de leurs paroisses.

Le deuxième invariant est le narrateur incarné par un garçon de messe. Dans le roman de Mongo Beti, il s'agit d'un des deux boys de la mission de Bomba : «Oui, nous sommes deux boys à Bomba, mais Daniel est mon aîné de plus de quatre ans... » (Mongo Beti, 2011 : 21). Plus avant, dans le texte, le lecteur découvre son nom : «- Denis, m'a-t-il dit, vous devriez être partis, le cuisinier et toi... » (Mongo Beti, $2011: 31$ ).

Le narrateur reste donc anonyme assez longuement avant d'être identifié par le RPS lui-même, alors que dans celui de Sender, son nom ne sera jamais dévoilé. Il ne sera désigné que par son rôle dans l'église, le « monaguillo ", le garçon de messe :

Le garçon de messe entrait, prenait une cloche qui se trouvait dans un coin, et, la maintenant bien soigneusement plus bas pour l'empêcher de sonner, il allait sortir quand Mosén Millán lui demanda :- Les parents sont-ils là ? (Sender, 1999 : 9) ${ }^{3}$

Donc, au-delà de leur dissemblance, ces deux romans présentent des ressemblances à des niveaux fondamentaux : leur thématique et le statut de leur narrateur. Le troisième niveau est celui de leurs titres.

\section{Titrologie des romans}

Rappelons que "la titrologie est la discipline qui s'intéresse à l'étude des titres d'œuvres artistiques ou journalistiques» (Ovono Ebè, 2007 : 69). Depuis sa conception par Léo Huib Hoek en 1981, la titrologie a fait l'objet de nombreuses recherches. Ainsi Michel Bernard la définit comme "une discipline de l'histoire littéraire qui réduit son champ à 
l'étude des titres d'œuvres » (1995: 135-141). Elle ne s'écarte pas des autres disciplines scientifiques du fait qu'elle offre une série d'outils pour une étude minutieuse des titres des œuvres.

La titrologie des deux romans de notre corpus peut se faire autour de deux aspects principaux : la micro-grammaticalité et la rhétorique.

6 La micro-grammaticalité fait apparaître deux titres grammaticalement différents, malgré les cinq éléments qui les composent: /Le/ pauvre/ Christ/ de/ Bomba/ - / Réquiem/ por/ un/ campesino/ español/.

L'étude de la micro-grammaire est rendue possible grâce aux «formes » qui « sont les "mots-outils" avec lesquels un auteur élabore un intitulé a priori adapté à son texte. Il s'agit d'articles, de pronoms, de conjonctions, d'interjections et de verbes conjugués ou non » (Ovono Ebè, $2007: 69$ ).

Michel Bernard dénombre treize « formes » les plus fréquentes dans l'histoire littéraire française entre les années 880 et 1991 ; ce sont essentiellement des articles, des conjonctions et des pronoms : de, la, le, les, l', et, du, des, d', ou, un, en, à.

Si toutes les "formes" retrouvées dans Le pauvre Christ de Bomba (le et de) sont comprises dans cette liste, il n'en est pas de même pour Réquiem por un campesino español - Requiem pour un paysan espagnol - dont la «forme » pour est absente de la liste. Cette absence est significative de la non exhaustivité de la liste de Michel Bernard. Car, en plus de pour, on peut aussi ajouter par, ce, ces, cet, cette, dont, sa, son, ses, mais, leur, leurs, je, tu, il, nous, vous, ils, me, te, se. On passerait ainsi de treize à vingt-deux "formes " micro-grammaticales, voire plus. Mais de toutes ces «formes", seules le, de, pour et un nous intéressent du fait de leur présence dans les titres qui font l'objet de notre étude.

$L e$ et de sont les «formes" présentes dans le titre de Mongo Beti. Ce sont un article et une préposition qui ont tout leur sens déictique au même titre que pour et un dans le titre de Ramón J. Sender.

Dans Le pauvre Christ de Bomba, l'article défini le détermine un nom propre (Christ) précédé d'un adjectif (pauvre), alors que la préposition de introduit le complément circonstanciel de lieu qui peut à la fois désigner l'origine ou l'appartenance (Bomba). Ce pauvre Christ peut donc être originaire de Bomba ou appartenir à Bomba, selon les cas tout au long du récit.

Pour ce qui est des "formes" dans Réquiem por un campesino español, pour est une préposition qui sert à désigner l'âme à l'intention de laquelle le requiem est dit ou doit être dit (le défunt Paco), alors que l'article indéfini un désigne un espagnol en particulier (Paco) parmi d'autres espagnols.

Le, de, pour et un sont donc les mots-outils qui forment la micro- grammaire des deux titres. Mais cette micro-grammaire n'est pas dénuée de sens dans la mesure où elle prépare à la rhétorique des titres: "La théorie de la rhétorique des titres permet d'aller audelà des seules formes micro-grammaticales pour mieux s'imprégner de la quintessence des énoncés du roman » (Ovono Ebè, $2007: 87$ ).

En étudiant la rhétorique des titres chez Montaigne sur la base des travaux de Pierre Villey, Claudie Balavoine et Hugo Friedrich, Luc Vaillancourt offre différentes pistes pour une lecture pertinente du rapport du titre avec son texte et une typologie des titres de romans selon leurs orientations discursives. De cette classification se dégagent six formes de titres: "fourre-tout ", "sentence ", "réfractaire ", " périphrastique ", «bouclier » et « écran »; l'auteur rappelle que les notions de " titre périphrastique » et de « titre bouclier » sont de lui. 
De ces six formes de titres, celles qui nous intéressent sont le «titre fourre-tout » et le «titre périphrastique ».

Luc Vaillancourt pense que "le titre fourre-tout autorise tous les détours et permet de jeter sous lui, pêle-mêle, une succession d'anecdotes des plus disparates » (Vaillancourt, 1994 : 41), et du titre périphrastique qu'il « tourne autour du sujet, sans jamais mentir mais sans trop en dire non plus » (Vaillancourt, 1994 : 41). Qu'il s'agisse de Le pauvre Christ de Bomba ou de Réquiem por un campesino español, nous pouvons dire que ce sont des titres à la fois «fourre-tout» et "périphrastiques»: ils autorisent «tous les détours » et "permettent de jeter sous eux» "pêle- mêle, une succession d'anecdotes des plus disparates", mais aussi qu'ils tournent "autour du sujet, sans jamais mentir mais sans trop en dire non plus » (Vaillancourt, 1994 : 41). Ainsi, avec Réquiem por un campesino español, on peut se demander s'il y a vraiment eu requiem. Et si oui, dans quelles conditions cette messe a-t-elle été proférée. Il en est de même pour Le pauvre Christ de Bomba autour duquel qui « jette sous lui » de petites histoires aussi bien sur la sociogenèse de Mongo Beti, son auteur, que sur la création romanesque et la construction des personnages du roman. « Ne jamais mentir sur le sujet et ne pas trop en dire non plus » est justement la caractéristique du titre Le pauvre Christ de Bomba selon que Ida Tomas qui affirme à ce sujet que

la terminologie hésite entre le concept et l'affect, qui distille ses dissonances à travers

l'accolement disparate des termes, et ce, dans un climat psychologique déjà quelque peu procédurier et néanmoins passionnel... Signification pléthorique dans un emploi polémique et déconcertant en raison de la problématique attachée au personnage qui donne nom et corps au texte: le Christ, créature extra-ordinaire, héros, homme particulier ou symbole!

(Tomas, $2008: 45$ )

Cette hésitation et surtout cette signification pléthorique conduisent le titre à « ne pas mentir et à ne pas trop en dire non plus " sur le ou les sujets du roman. C'est sur cela que repose la rhétorique des titres : permettre d'accorder ces derniers aux textes dont ils constituent l'intitulé. Mais le rapport entre le titre et le texte ne peut être clairement défini sans résoudre le problème de la date d'édition.

La date d'édition est tellement importante qu'elle perturbe l'antériorité ou la postériorité d'un roman. Si le problème ne se pose pas pour Le Pauvre Christ de Bomba dont le titre n'a jamais été influencé par la date, quelles que soient les éditions, ce n'est pas le cas pour Réquiem por un campesino español. En effet, depuis la première édition de 1956, le Camerounais n'a jamais changé le titre de son roman, contrairement à l'Espagnol qui a d'abord publié son texte sous le titre Mosén Millán en 1953, avant d'en lancer une autre édition en 1960 sous le titre qui sera désormais retenu : Réquiem por un campesino español. Selon Borja Rodríguez, Sender a justifié ce changement de titres en ces termes: "En publiant l'édition nord-américaine en 1960, il se trouva que le titre originel n'avait aucun sens pour ce public: "en anglais, il ne rimait à rien" (Rodríguez Gutiérrez, $2012: 338)^{4}$.

Il est donc difficile de déterminer la date exacte de publication de ce texte à cause de cette variation des titres. Prendre en considération le titre Mosén Millán fait de Ramón J. Sender un précurseur de la pensée de Mongo Beti quant à l'image du prêtre dans la société. Par contre, le titre Réquiem por un campesino español érige le Camerounais en avant-gardiste par rapport à l'Espagnol.

Pour ne pas alimenter la polémique sur ces dates d'édition, nous allons considérer le titre de l'édition de 1960, même si celui de 1953 aurait facilité une titrologie comparée des deux œuvres, ne serait-ce que dans leur aspect éponyme. Les personnages 
éponymes aurait été un des invariants dans cette approche comparée des deux titres : Mosén Millán, dans le roman de Ramón J. Sender et le père Drumont, Christ de Bomba, dans celui de Mongo Beti. Toutefois, la comparaison reste possible, notamment dans l'annonce par les deux titres de thèmes chrétiens. En d'autres termes, avec ces titres, le lecteur s'attend à des représentations chrétiennes. Le premier, Le pauvre Christ de Bomba, est plus clair à ce sujet, car, dès le début du roman, la présence d'un Christ à la mission de Bomba, non pas Jésus Christ, mais un prêtre qui lui ressemblait trait pour trait, est explicite :

"Dis-leur donc que Jésus Christ et le R.P.S. c'est tout un", lorsque les petits enfants de notre village, contemplant l'image qui représente le Christ entouré de gosses, furent étonnés par la ressemblance avec le R.P.S. : même barbe, même soutane, même cordon au niveau de la ceinture, et qu'ils s'écrièrent: "Mais! Jésus Christ... on dirait le R.P.S. !" Et mon père leur assura que Jésus et le R.P.S., c'est tout un. Et depuis lors, les gosses de mon village appellent le R.P.S. "Jésus Christ". (Mongo Beti, 2011 : 11)

Donc, parler du Christ de Bomba ne revient pas à signifier que Jésus Christ aurait séjourné à Bomba, mais qu'il y a une forte ressemblance entre Jésus Christ et le prêtre affecté à Bomba, aux niveaux de la prosopographie et de l'éthopée. Le père Drumont ne dit-il pas, lui-même, à Denis : "Je te dis que je suis comme le Christ : moi aussi, je pardonne toujours " (Mongo Beti, 2011 : 223). Ce sont donc là des évocations de la chrétienté aussi significatives que celle dont fait état le titre de Ramón J. Sender: Réquiem por un campesino español. Dans la religion catholique, le requiem désigne la prière en latin de l'office des morts. En substituant Réquiem por un campesino español à Mosén Millán, Sender a déplacé la focalisation du personnage éponyme vers la cérémonie éponyme ; car le roman repose précisément sur la messe de requiem que Mosén Millán doit dire pour l'âme du défunt Paco en présence de ses parents, amis et connaissances :

Esperaba que los parientes del difunto acudirían. Estaba seguro de que irían - no podían menos - tratándose de una misa de réquiem, aunque la decía sin que nadie se la hubiera encargado. También esperaba Mosén Millán que fueran los amigos del difunto. (Sender, $1999: 8)^{5}$

Refuser le caractère éponyme au prêtre, en 1960, sept ans après la première édition (1953), revient à accorder plus d'importance à la messe de requiem elle-même qu'au prêtre qui doit la dire. C'est donc un changement de perspective qui va du sentiment de culpabilité de Mosén Millán à la tragédie de Paco, comme le démontre Borja Rodríguez Gutiérrez : «El cambio de título implicaba además un cambio de perspectiva: del sentimiento de culpa de mosén Millán a la tragedia de Paco el del Molino, los dos polos entre los que oscila todo el relato " (Rodríguez Gutiérrez, $2012: 238)^{6}$. Requiem pour un paysan espagnol est ainsi un titre qui indique clairement qu'il va s'agir de la narration d'une messe de requiem tout au long du récit, même si celle-ci n'aura pas lieu dans les conditions espérées par Mosén Millán.

Nous pouvons conclure que ces deux titres sont éminemment chrétiens. Mais ces intitulés chrétiens annoncent-ils des attitudes christiques de la part du prêtre, qu'on soit dans le Cameroun peint par Mongo Beti dans Le pauvre Christ de Bomba ou dans l'Espagne décrite par Ramón J. Sender dans Réquiem por un campesino español ? Il faudrait explorer les agissements du curé aussi bien dans ses rapports avec le pouvoir politique et avec les fidèles dans les deux textes. 


\section{L'église et le pouvoir : histoire d'une interrelation certaine}

9 Aussi bien dans Réquiem por un campesino español que dans Le Pauvre Christ de Bomba, le pouvoir est représenté dans ses différentes sphères: économique, administrative et militaire.

Dans Réquiem por un campesino español, le pouvoir administratif est représenté par le maire (el alcalde) (Sender, 1999: 64), la mairie (el ayuntamiento) (Ibidem: 78), les municipalités (los municipios) (Ibidem : 70) et le duc (el duque) (Ibidem : 68); le pouvoir militaire l'est au travers de "la guardia civil de la aldea [...] un grupo de señoritos con vergas $y$ con pistolas " (Ibidem : 81$)^{7}$ et du centurion.

Les trois personnalités les plus puissantes du village incarnent le pouvoir à la fois économique et administratif. Il s'agit de don Valeriano, don Gumersindo et señor Cástulo: "...las dos familias más pudientes: don Valeriano y don Gumersindo. La tercera familia rica, la del señor Cástulo Pérez... » (Ibidem : 9) ${ }^{8}$. Parmi les trois, don Valeriano est le plus proche du pouvoir administratif de par son statut d'administrateur: «...don Valeriano [...] el administrador » (Ibidem : 71-76) ${ }^{9}$.

La même configuration ostensible du pouvoir est lisible dans Le Pauvre Christ de Bomba, avec une spécificité du pouvoir administratif: il est représenté par un jeune administrateur Vidal, plus actif que son vis-à-vis dans le roman de Sender.

Les multiples entretiens de ce dernier avec le père Drumont témoignent de cette omniprésence du pouvoir administratif dans la mission évangélisatrice. Il fait son entrée dans le récit à Kota, troisième étape du R.P.S., lors de sa "tournée au pays des Tala» (Mongo Beti, 2007 : 14). Lors de cette étape, il a la particularité de procurer une certaine euphorie au prélat :

Il semble rêveur, plus confiant, et il s'amuse un peu de tout ce que lui rapporte le catéchiste.

Il a cet aire-là depuis la conversation qu'il a eue avec le jeune administrateur, un plaisantin quil'a bien fait rire. (Mongo Beti, $2007: 57$ )

C'est d'ailleurs au cours de l'un de ces entretiens que le lien étroit entre le pouvoir administratif et l'église est évoqué par Vidal: "[...] ma sublime vocation, une vocation unique... hum ! avec la vôtre, quoi ! D'ailleurs, je continue à soutenir et contre vous que la mienne et la vôtre n'en font qu'une... » (Mongo Beti, 2007 : 58). C'est donc l'affirmation d'un destin commun entre ces deux entités. Mais à ces deux entités, l'administration et la mission évangélisatrice, il faut aussi ajouter une troisième, le commerce ou l'activité économique, pour parfaire ce que Philippe Basabose nomme le «triumvirat colonial » (Basabose, $2008: 34$ ), c'est-à-dire missionnaire, armée/administration, commerçant.

Il en est de même dans le roman de Ramón J. Sender, malgré la différence contextuelle. Certes, Mongo Beti dépeint l'Afrique sous la colonisation européenne, alors que Sender décrit l'Espagne pendant la guerre civile. Mais le mécanisme reste étonnamment le même, la même complicité entre les trois composantes du pouvoir déjà présentes dans Le Pauvre Christ de Bomba. Cette constance du rapport entre le curé et le pouvoir, qu'il soit administratif ou économique, se révèle comme une liaison dangereuse. Car elle met en péril le rapport entre le curé et le fidèle. Ainsi est posée la question du rapport entre le prêtre et ses fidèles. 


\section{Drumont et Mosen Millan : prêtre ou antéchrist?}

10 On parle d'antéchrist pour désigner cette figure symbolique, à l'opposé du Christ, qui représente les forces hostiles à Dieu. Dans les textes de Mongo Beti et de Sender, l'antéchrist est le prêtre dont les agissements vont à l'encontre des principes chrétiens.

Si les deux romanciers choisissent de conduire la narration à travers un personnage religieux - le jeune et naiff Denis ( 15 ans) pour Mongo Beti et le monaguillo ( 7 ans) pour Sender - , c'est pour pouvoir créer un décalage entre la réalité prosaïque et un certain idéal chrétien, qu'on soit en pleine colonisation ou en pleine guerre civile espagnole dont le roman de Sender est une représentation symbolique, allégorique, selon Borja Rodríguez Gutiérrez :

Una representación simbólica de la guerra civil española, de sus causas y de sus consecuencias, de los elementos principales y de los agentes del conflicto. En este sentido se puede definir el Réquiem por un campesino español como una cuidada y completa alegoría. (Gutiérrez, $2012: 340)^{10}$

Et c'est cet idéal qui amène le berger, non plus à protéger ses brebis, mais à les livrer. C'est alors qu'intervient la trahison. Elle s'exprime diversement selon Le Pauvre Christ de Bomba ou Réquiem por un campesino español. Dans le roman de Mongo Beti, la trahison résulte de l'absolue nécessité du Père Drumont d'évangéliser le maximum d'Africains possible, peu importe la manière par laquelle il doit y arriver. Et, l'un des moyens les plus sûrs de se faire d'y parvenir est la maltraitance ou les humiliations pendant les travaux de construction routière. On peut trouver plusieurs autres exemples dans le texte, mais celui qui semble le plus expressif est cette tirade du RPS lors de son entretien avec l'administrateur Vidal à son étape de Kota :

Je vais vous faire une confidence: il n'y a que les gens de la route pour être bons chrétiens

[...] Les gens de la route vivent dans une terreur constante [...] Ils vivent dans une terreur perpétuelle à cause des réquisitions, des travaux forcés, des bastonnades, des tirailleurs... Croient-ils réellement, ou se tournent-ils vers moi qui les console - sans jamais d'ailleurs pouvoir les protéger? (Mongo Beti, $2007: 62$ )

Cette tirade est absolument fondamentale dans l'œuvre parce qu'elle explique la trahison du prêtre. En effet, pour le Père Drumont, la fin justifie les moyens. La logique du chiffre conduit le curé à livrer le troupeau qu'il devait protéger pour que ce dernier trouve refuge chez lui. C'est l'aveu d'impuissance du seul discours évangélique quant à l'objectif de christianiser les peuples sous la colonisation. Donc, au moment où les peuples s'attendent à être protégés par l'Église contre tous les abus coloniaux, cette dernière encourage plutôt ces atrocités en vue d'avoir de la "clientèle » (Mongo Beti, 2011 : 61), pour parler comme l'administrateur Vidal. Sous cet angle, le R.P.S est aussi antéchrist que le sorcier Sanga Boto qu'il considère comme «l'incarnation de Satan [...] venu dans ce pays pour tromper les chrétiens, les enfants de Dieu» (Ibidem: 129). Il y a une sorte de conspiration de l'Église et du pouvoir colonial contre les peuples à évangéliser.

La même trahison et la même conspiration sont présentes dans le roman de Sender, avec la représentation de l'Espagne à travers l'histoire a lieu dans un petit village, du peuple espagnol avec Paco el del Molino, de l'église espagnole avec Mosén Millán, le pouvoir économique avec Don Valeriano et Don Gumersindo (propriétaires terriens) et El Sr. Cástulo (la bourgeoisie financière). Ce symbolisme est d'une importance capitale dans la compréhension du mécanisme de la trahison dont le processus commence au moment de la réforme agraire préconisée par Paco. Celui-ci décide de ne plus payer 
l'impôt foncier au Duc, propriétaire terrien. Mosén Millán, Don Valeriano et Don Gumersindo se réunissent alors pour préparer une réponse énergique à cet insoumis.

El Sr Cástulo qui souhaite aussi se joindre à eux se voit opposer une fin de non-recevoir. Les autres représentants du pouvoir manquent de confiance en lui car ils ne cernent pas ses intentions. Et pourtant il est bien des leurs, puisqu'il fait cause commune avec les troupes qui viennent installer Don Valeriano comme maire du village.

Commence alors le règne de la terreur dans le village : le cordonnier, qui symbolise la libre expression, est assassiné ; le médecin, qui incarne la science, est incarcéré, etc. Dans ce climat, Mosén Millán se réfugie à l'intérieur de son église et ne fait rien pour protéger le peuple. Sa seule préoccupation est que tous ces assassinats aient lieu sans laisser le temps aux victimes de se confesser. Mais il sort de sa retraite car sa collaboration est requise pour faire disparaître Paco et contribuer ainsi à la victoire de don Valeriano. Il trahit donc Paco et le livre à l'armée afin qu'il soit exécuté. Que l'église soit vide au moment de célébrer la messe de requiem n'est pas étonnant puisque le peuple n'a pas oublié la trahison. La messe elle-même est trahison parce qu'elle se fait contre de l'argent : il a fallu payer pour qu'elle ait lieu. C'est El Señor Cástulo qui nous informe de son coût: dix pesetas multipliés par trois (Don Valeriano, Don Gumersindo et el Señor Cástulo) pour atteindre la somme de trente pesetas pour rappeler celle des trente pièces de monnaie que le Grand Prêtre remit à Judas pour livrer Jésus. C'est ainsi que, par association, Mosén Millán, l'apôtre du Christ, devient Judas, l'incarnation de l'antéchrist, et les pharisiens seraient Don Valeriano, Don Gumersindo et El Señor Cástulo.

12 La religion, dans son rapport ambigu avec le pouvoir, est bien le thème central dans les deux textes et l'étude titrologique le prouve. Mongo Beti le confirme lui-même dans la revue Peuple Noir: "l'interrogation sur l'évangélisation missionnaire comme auxiliaire de l'asservissement des Africains est le sujet même du "Pauvre Christ de Bomba " (Mongo Beti, 1981 : 104-132). C'est la même interrogation dans Réquiem por un campesino español. Celle-ci est lisible dans les souvenirs de Mosén Millán qui facilitent la lecture de l'œuvre en même temps qu'ils donnent l'impression d'un véritable examen de conscience, comme l'affirme José Luís Negre Carasol :

Este devenir ordenado de los recuerdos de Mosén Millán es un elemento que añade la verosimilitud al relato, facilita la lectura de la obra y, además, da la impresión de constituir un 'examen de conciencia' del cura. (Negre Carasol, $1983: 58)^{11}$

Le Père Drumont est soumis au même examen de conscience avant de constater son échec et d'avouer à Vidal qu'il quittait désormais l'Afrique pour rentrer en France : "Bonne chance, mon petit Vidal! Moi, voyez- vous, je suis un vaincu. Je doute qu'on soit jamais allé plus loin dans la défaite [...] Je crains que je ne m'en aille vraiment. Sans espoir de retour» (Mongo Beti, 2007 : 244).

Ce constat d'échec aussi bien chez le RPS que chez Mosén Millán est aussi l'aveu d'une traitrise. Ainsi, aussi bien dans Le Pauvre Christ de Bomba que dans Réquiem por un campesino español, le prêtre n'est en définitive qu'un traitre pour le peuple qu'il est censé protégé : il choisit d'être de connivence avec le pouvoir pour trahir le peuple tout en tentant de le gagner.

Finalement, comme Ramón J. Sender, Mongo Beti « a consacré sa vie d'homme et d'écrivain à dénoncer les forces qui font le malheur des peuples et à identifier celles de la répression contre l'émancipation " (Mambenga, 2008 : 9). Réquiem por un campesino español et Le Pauvre Christ de Bomba, à travers le parcours narratif du prêtre, la réactualisation du débat sur les 
rapports entre l'Église et l'État le démontrent, et de ce fait, peuvent être considérés comme des romans engagés.

\section{BIBLIOGRAPHIE}

BASABOSE P., « Mongo Beti : l'art du polémotexte », in MAMBENGA Frédéric (dir), Interculturel Francophonies $\mathrm{n}^{\circ}$ 13, Mongo Béti : la pertinence réaliste et militante, juin-juillet 2008, p. 31-43. BERNARD M., « À juste titre : Une approche lexicométrique de la titrologie. » Article paru en anglais sous le titre « À juste titre : a lexicometric Approch to the Study of Titles », in Literary and Linguistic Computing, vol. 10, $\mathrm{n}^{\circ} 2$ 2, 1995, p. 135-141.

BEYALA C., Le Christ selon l'Afrique, Paris, Albin Michel, 2014.

MAMBENGA F., et al, Interculturel Francophonies $n^{\circ} 13$, Mongo Beti : la pertinence réaliste et militante, Lecce, Alliance Française, 2008.

MONGO BETI, «Le Pauvre Christ de Bomba expliqué », in Peuples Noirs Peuples Africains n 19, 1981, 104-132.

MARINO A., «Etiemble, la typologie des invariants et la littérature comparée », in Canadian Review of Comparative Literature, VI, $\mathrm{n}^{\circ} 4$ (Fall 1979), p. 323-345.

MONGO BETI, Le Pauvre Christ de Bomba, Paris, Présence Africaine, 2011.

NEGRE CARASOL J.L., « Analepsis en Réquiem por un campesino español de Ramón J. Sender », in Argensola : Revista de Ciencias Sociales del Instituto de Estudios Altoaragoneses, 1983, p. 53-68. OVONO EBE M., L'enfant dans le roman espagnol de la première décennie de la l'après-guerre civile (1939-1952) : Complexité des relations entre l'enfant et son environnement, Thèse de doctorat Nouveau Régime, Montpellier, Université Paul-Valéry - Montpellier III, 2007.

RODRÍGUEZ GUTIÉRREZ B., « Reiteración y simbolismo en "Réquiem por un campesino español” de Ramón J. Sender », in Boletín de la Asociación de profesores de Español "Gerardo Diego", núm. 11, 2005, pp. 10-19.

RODRÍGUEZ GUTIÉRREZ B., « Un planteamiento didáctico del Réquiem por un campesino español de Ramón J. Sender », In Didáctica, Lengua y Literatura, vol. 24, 2012, p. 337-361.

SENDER R. J., Réquiem por un campesino español, Barcelone, Destino, 1999.

TOMAS I., «Fornication et évangélisation... ou l'art d'aimer chez Mongo Béti », in MAMBENGA Frédéric (dir), Interculturel Francophonies $\mathrm{n}^{\circ} 13$, Mongo Béti : la pertinence réaliste et militante, juinjuillet 2008, p. 45-59.

VAILlAnCoURT L., Titrologie des Essais : vers une poétique de l'informe, Montréal, Mémoire de Maîtrise, Université Mc Gill, 1994. 


\section{NOTES}

1. Calixte Béyala est une romancière franco-camerounaise. Elle a publié Le Christ selon l'Afrique en 2014.

2. Traduction en français : « Requiem pour un paysan espagnol ».

3. "Le garçon de messe entrait, prenait une cloche qui se trouvait dans un coin, et, la maintenant bien soigneusement plus bas pour l'empêcher de sonner, il allait sortir quand Mosén Millán lui demanda: - Les parents sont-ils là?».

4. "En publiant l'édition nord américaine en 1960, il se trouva que le titre originel n'avait aucun sens pour ce public : "en anglais, il ne rimait à rien". »

5. Traduction en français: "Il attendait l'arrivée des parents du défunt. Il était sûr qu'ils viendront - ils n'en pouvaient pas moins - puisqu'il s'agissait d'une messe de requiem, même s'il la disait sans que personne ne le lui demande. Mosén Millán s'attendait aussi à ce que les amis du défunt viennent. "

6. Traduction en français: «Le changement de titre impliquait, en plus, un changement de perspective : du sentiment de culpabilité de Mosén Millán à la tragédie de Paco el del Molino, les deux pôles entre lesquels oscille le récit.»

7. Traduction en français: «La gendarmerie du village [...] un groupe d'hommes munis de vergues et de pistolets. »

8. Traduction en français: "...Les deux familles les plus puissantes: don Valeriano y don Gumersindo. La troisième famille riche, celle de monsieur Cástulo Pérez... »

9. Traduction en français : «...don Valeriano [...] l'administrateur ».

10. Traduction en français : «Une représentation symbolique de la guerre civile espagnole, de ses causes et de ses conséquences, des éléments principaux et des agents du conflit. Ainsi peut-on définir Réquiem por un campesino español comme une attentive et complète allégorie. »

11. Traduction en français : «Ce devenir ordonné des souvenirs de Mosén Millán est un élément qui ajoute de la vraisemblance au récit, facilite la lecture de l'œuvre et, en plus, donne l'impression de constituer un 'examen de conscience' du curé. »

\section{RÉSUMÉS}

Cette contribution vise à croiser le comportement de l'Église, aussi bien lors de la colonisation française en Afrique que pendant la Guerre Civile en Espagne, à travers deux textes : Le Pauvre Christ de Bomba (1956) de Mongo Beti et Réquiem por un campesino español (1960) de Ramón J. Sender ; deux romans qui font de l'Église leur personnage principal. Les points de comparaison sont essentiellement le thème de la religion autour du personnage du prêtre, la posture du narrateur incarné par le garçon de messe et le paratexte avec une priorité accordé aux titres. Nous concluons sur l'actualité de la question que posent ces deux romans et qui toujours débat, à savoir la séparation de l'Église et de l'État.

This contribution aims at crossing the behavior of the Church during the French colonization in Africa and during the Civil War in Spain, through Le Pauvre Christ de Bomba (1956) by Mongo Beti and Réquiem por un campesino español (1960) by Ramón J. Sender. The points of comparison are essentially the theme of the religion around the character of the priest, the posture of the narrator embodied by the boy of mass and the paratext with a priority granted to the titles. We 
conclude on the relevance of the question posed by these two novels and which is still debate, namely the separation of Church and State.

INDEX

Mots-clés : Le Pauvre Christ de Bomba, Réquiem por un campesino español, Mongo Beti, Ramón J. Sender, analyse thématique

Keywords : Le Pauvre Christ de Bomba, Réquiem por un campesino español, Mongo Beti, Ramón J. Sender, thematic analisis

\section{AUTEUR}

\section{EBÉ MATHURIN OVONO}

Université Nationale du Gabon Libreville - Gabon 